\title{
The Common Airborne Instrumentation System Program Overview
}

\author{
Sidney R. Jones, Jr. \\ CAIS Lead Engineer \\ Naval Air Warfare Center, Aircraft Division \\ Patuxent River, Maryland 20670
}

\begin{abstract}
The Common Airborne Instrumentation System (CAIS) is being developed by the Department of Defense through a Tri-service Program Office. The goals of the program are two fold. The first is to develop an instrumentation system that will meet the needs of the Air Force, Army, and Navy into the next century. The system is designed to support a full breadth of applications from a few parameters to engineering and management and development programs. The second is to provide a system that is airframe as well as activity independent.

To accomplish these goals, the CAIS consists of two segments. The airborne segment consists of a system controller with a suite of data acquisition units. The system is configured with only the units that are required. The ground segment consists of a variety of support equipment. The support equipment enables the user to generate formats, load/verify airborne units, perform system level diagnostics and more.
\end{abstract}

\section{KEY WORDS}

Airborne Instrumentation, Signal Conditioning, Data Acquisition

\section{INTRODUCTION}

Historically, the test ranges each had their own complement of instrumentation systems. For each new aircraft program there was always a new test requirement so the airframe contractors developed new instrumentation systems to achieve the new capability. This led to many instrumentation systems in the field. The costs associated with keeping personnel trained on each system, finding resources to keep the systems up and running and buying spares for the new systems was getting prohibitive. 
The CAIS Joint Program Office was created as a tri-service program to provide a flexible and expandable instrumentation system for the Department of Defense. By utilizing a common system, personnel need only be trained once and spare equipment is required for one system type vice many. Interoperability between ranges will be greatly increased due to the existence of ground support, emergency spares and technical expertise already there.

\section{AIRBORNE SEGMENT}

There are three major building blocks in a generic CAIS system: Overhead functions, Data Acquisition Units (DAUs) and signal conditioners. The overhead consists of an airborne controller which commands the data acquisition units and receives data over the CAIS bus. The controller also formats the data into an IRIG compatible PCM stream. The data acquisition unit can be designed for the collection of specific data or the collection of data from a variety of sources. Signal conditioning cards are designed to interface to specific signal types through the use of a general purpose DAU.

The CAIS is designed to be a distributed, modularly expandable data acquisition system. The user installs only the hardware that is essential at the required location eliminating the need to run large bundles of wires throughout the aircraft. As new requirements are identified, the system capability may be increased through reprogramming and adding DAUs when needed. If necessary, a new DAU can be designed and added to the existing system. The airborne controller commands the DAUs and receives data from the DAUs via the CAIS bus (figure 1). An airborne controller can utilize up to 3 buses per system. The CAIS bus is a hybrid star/daisy chain design which is implemented through the use of bus splitters to allow for flexibility in routing through the aircraft. Up to 60 DAUs can be placed on one bus with a maximum of 180 DAUs across 3 buses. The CAIS DAUs are completely programmable across the CAIS bus. The CAIS system can handle up to 8000 channels of selected data and time tags with a throughput rate from $2 \mathrm{Kbps}$ to $15 \mathrm{Mbps}$. With the addition of the PCM combiner, data rates up to $50 \mathrm{Mbps}$ can be achieved.

\section{Airborne System Controller (ASC)}

The ASC orchestrates the data collection for the system. The data received across the CAIS bus is formatted into several different outputs. The primary PCM stream (max bit rate $15 \mathrm{Mbps}$ ) is available in 3 output codes, NRZ-L, RNR-L and BI $\phi-\mathrm{L}$, simultaneously. There are 3 programmable secondary outputs that can be individually programmed for a subset of the PCM words in the primary PCM stream. The programmable secondaries can be programmed in any of the 3 output codes. There are up to 16 dedicated secondary outputs. They are "dealt" to the dedicated secondaries one word at a time. There is also a byte wide output for rotary head recorders. The 


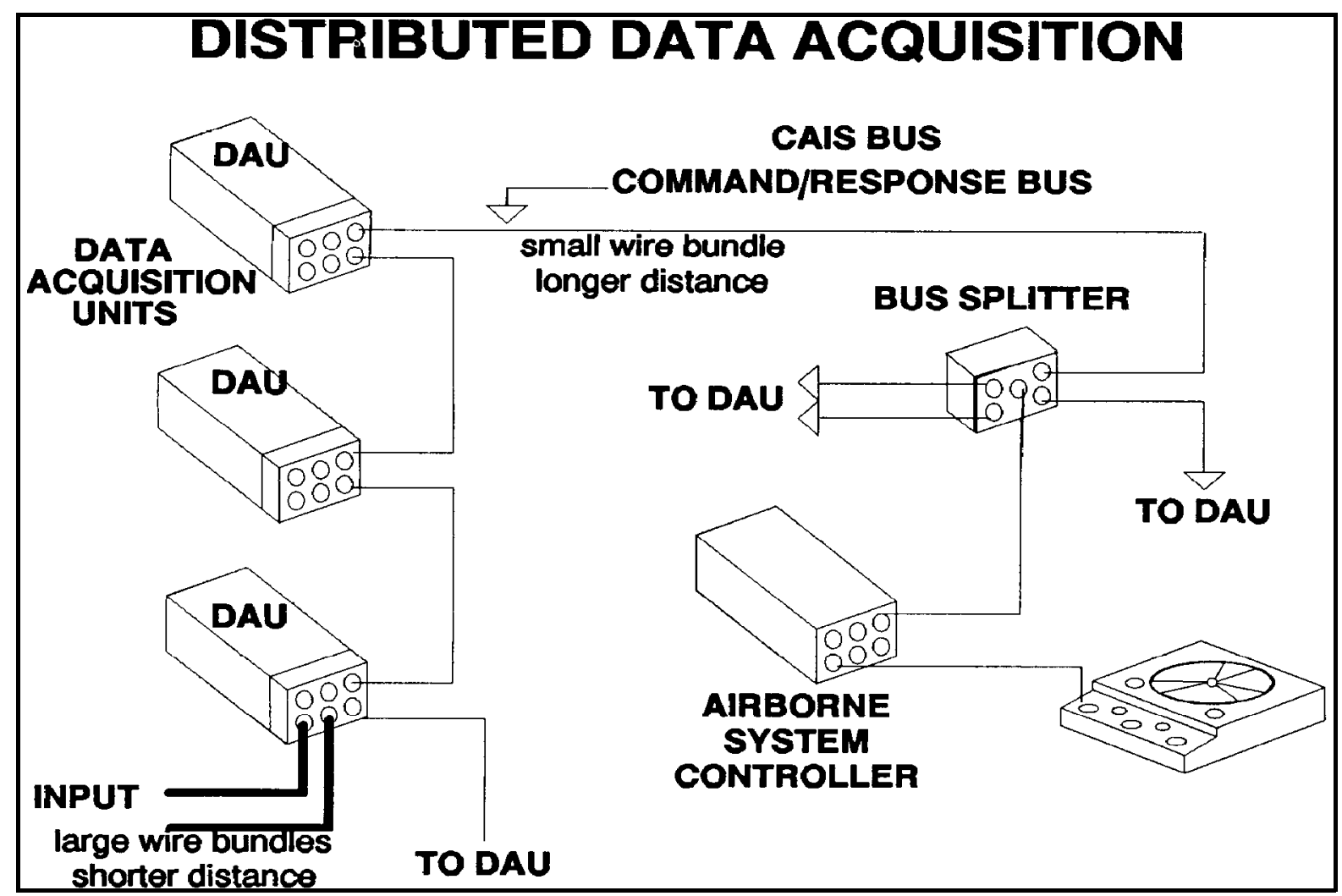

Figure 1

ASC can support up to 8 sampling formats in memory at once. There is an internal time code generator that will count elapsed time or can synchronize to an external source of IRIG modulated B or IRIG non-modulated A, B or G time. The ASC provides 2 modulated and 4 non-modulated outputs. There is an external Airborne Battery Pack (ABP) to prevent loss of time due to power interruptions. There are 3 additional capabilities that are added to the ASC when needed: The Airborne Processor (AP), the 1553RT and the PCM Combiner Slice (PCMC Slice). The AP performs engineering units conversions and other data manipulations. The AP provides a display interface for up to 16 data displays throughout the aircraft. The AP is user programmable and can store up to 8 programs in memory. The 1553RT operates as a remote terminal on the aircraft's 1553B avionics bus. This allows the user to place selected raw PCM or engineering units data on one or more of the aircraft's displays. The PCMC slice is a 4 input version of the PCM Combiner to be discussed next.

\section{$\underline{\text { PCM Combiner (PCMC) }}$}

The PCMC comes in two versions. The slice version attaches to the ASC and provides 4 inputs with a throughput of $50 \mathrm{Mbps}$. The standalone version provides 16 inputs with a throughput of $50 \mathrm{Mbps}$. In both configurations, NRZ-L data and bit rate clock are required as inputs. Each input is unsynchronized in relation to the other inputs. 
The output bit rate is selectable up to $55 \mathrm{Mbs}$. The PCM output is available as an Emitter Coupled Logic (ECL) output and as RS422 and their associated clocks. A byte wide output is also available at one eighth the bit rate of the serial output.

\section{Analog-Discrete Data Acquisition Unit (ADAU)}

The ADAU is a general purpose data acquisition unit. There are 18 different signal conditioning card types that support the ADAU. The ADAU has 10 general purpose signal conditioning slots available. The ADAU has a 12 bit A/D for analog samples. The ADAU has a throughput of 417 thousand samples per second. By using one of the signal conditioning slots the ADAU can run in standalone mode where it will generate a PCM stream with a bit rate of $39 \mathrm{Kbps}$ to $5 \mathrm{Mbps}$. The ADAU can also be used as a small system controller. ADAU signal conditioning cards include:

\begin{tabular}{|c|c|c|}
\hline Name & Ch/Card & Brief Description \\
\hline Analog Attenuator & $(8)$ & Inputs to $17 \overline{5 \text { volts }}$ \\
\hline \multirow[t]{2}{*}{ Analog Data Filter } & (4) & Low pass butterworth filter $2 \mathrm{~Hz}$ to $10 \mathrm{Khz}$ \\
\hline & (4) & Low pass bessel filter $120 \mathrm{~Hz}$ to $960 \mathrm{~Hz}$ \\
\hline ARINC 429 Monitor & (1) & $1 \mathrm{~K}$ word storage from ARINC 429 bus \\
\hline Auto Range Conditioner & (1) & Auto/Man gain to $1024 ; 60 \mathrm{~K}$ samples/sec max \\
\hline Control Signal Generator & (4) & Relay and TTL outputs to control external events \\
\hline Digitized Audio Conditioner & (1) & CVSD encoding; 16 to $35 \mathrm{Kbps}$ \\
\hline Event Time Recorder & (7) & Time correlation of external events to $0.01 \mathrm{~ms}$ \\
\hline Extended Multiplexer & (12) & $\begin{array}{l}\text { Direct analog inputs with gain and offset per } \\
\text { channel }\end{array}$ \\
\hline Freq Converter/Pulse Tot. & (4) & Frequencies $5 \mathrm{~Hz}$ to $256 \mathrm{KHz} ; 32$ bit pulse count \\
\hline 1553 Transducer Interface & (1) & Transducer 1553 bus controller; 8 messages \\
\hline Parallel Digital Cond. & (16) & Same input characteristics as the DDAU \\
\hline Phase Sensitive Demodulator & (1) & $20 \mathrm{~Hz}$ to $20 \mathrm{KHz}$ excitation \\
\hline Serial Digital Cond. & (1) & Synchronous and Asynchronous cards available \\
\hline Simultaneous Sample Cond. & (7) & Gain, offset, 12 bit A/D \\
\hline Synchro/ResolverCond. & (2) & 15 bit resolution, $360 \mathrm{~Hz}$ to $1 \mathrm{Khz}$ \\
\hline Thermocouple Signal Cond. & (7) & Type J,K,E,T; External ref. \\
\hline Transducer Excitation Supply & (4) & Programmable voltage source \\
\hline Variable Resistance Sensor & (4) & Programmable current source with conditioning \\
\hline
\end{tabular}

\section{Miniature Data Acquisition Unit (MDAU)}

The MDAU is another general purpose data acquisition unit. It is an Aydin Vector MMSC-800 unit with a CAIS bus interface. The small size of this unit allows the MDAU to be placed in small hard to reach areas through out the aircraft. The initial capability of the MDAU is for 5 selected signal conditioning modules. However, any of the signal conditioning modules available for the MMSC-800 will work on the CAIS version. The MDAU is based upon the 'slice' concept where every signal conditioning module added increases the size of the unit. Up to 31 modules can be 
used on each MDAU. The MDAU cannot be used in a stand alone mode and remain programmable over the CAIS bus. The following are the current CAIS supportable MDAU signal conditioning modules.

\begin{tabular}{lcl} 
Name & Ch/Card & \multicolumn{1}{c}{ Brief Description } \\
Bridge Interface & $(2)$ & Programmable gains \\
Direct Multiplexer & $(8)$ & Differential Inputs \\
Synchro/Resolver & $(1)$ & 16 bit resolution \\
Discrete Interface & $(24)$ & 12 bits/word, TTL inputs, single ended \\
Frequency to Digital & $(2)$ & 12 bit resolution, can be cascaded for 24 bit resolution
\end{tabular}

\section{Avionics Data Acquisition Unit (AVDAU)}

The AVDAU consists of a programmable core unit that will monitor 1553, H009 or the F-16 Weapons bus. In addition, there are Bus Interface Modules (BIM) that will expand the 1553 capability of the core unit. The core unit can monitor 2 dual redundant 1553 buses, 2 dual redundant $\mathrm{H} 009$ bus, 1 dual redundant 1553 and 1 dual redundant $\mathrm{H} 009$ or the Weapons bus half duplex plus dual simplex. Each bus interface module can monitor an additional 2 dual redundant 1553 buses. The maximum AVDAU configuration is a core unit with 3 BIMs. Selected data from any of the buses for multiple formats is handled in one message list. The user may select up to a maximum of 4096 messages per bus with a total of 32,000 words, any or all of which may be time tagged. The AVDAU is IRIG 106-93 chapter 8 compatible for $100 \%$ bus data acquisition and provides these outputs simultaneous with the selected data. There are 16 discrete inputs on the core unit which may be included in the selected data output. The AVDAU can also work in a standalone mode. It can have up to 4 different formats with selected data output rates from $2 \mathrm{Kbps}$ to $5 \mathrm{Mbps}$ in addition to the $100 \%$ data.

\section{Discrete Data Acquisition Unit (DDAU)}

The DDAU is a compact unit designed for acquiring digital signals. There are 8 words of 16 bits each for a total of 128 discrete inputs. Each channel may be independently set up for single or differential operation and current or voltage mode. The 8 channels are divided into 3 groups for simultaneous sampling or external latching selection. The DDAU may be placed in stand alone mode where it will handle 7 formats with a PCM output rate of $7.8 \mathrm{Kbps}$ to $1 \mathrm{Mbps}$.

\section{Global Positioning System Data Acquisition Unit (GDAU)}

The GDAU operates from the instrumentation port on the tactical GPS receiver. It will receive the generic data or request specific data from the satellite. The GDAU converts the satellite time information to an IRIG A, B, or G output for supplying time to the ASC or other DAUs. There are 16 discrete inputs which are similar in 
characteristics to the DDAU. The GDAU can run in standalone mode where it can support up to 8 formats with an output rate of $7.8 \mathrm{Kbps}$ to $1 \mathrm{Mbps}$.

\section{SYSTEM CONFIGURATIONS}

The CAIS handles large system requirements, small system requirements and everything in between. To utilize the full capabilities of CAIS would require the use of an ASC and a PCM combiner. The large system can accommodate 180 DAUs with 8000 parameters into $15 \mathrm{Mbps}$ of selected data (figure 2). The PCM combiner (either as a slice or stand alone) would merge the ASC output, the $100 \%$ avionics bus data and several DAUs in stand alone mode into a single composite stream. If all that is required is PCM stream from several DAUs of $5 \mathrm{Mbps}$ or less, the ADAU could be used as a small system controller. The small system wouldn't force the user to have a separate controller. The ADAU would act as controller for up to 60 DAUs while collecting data itself. For smaller systems yet, when all the data is coming from a single DAU, the DAU would be operated in stand alone mode.

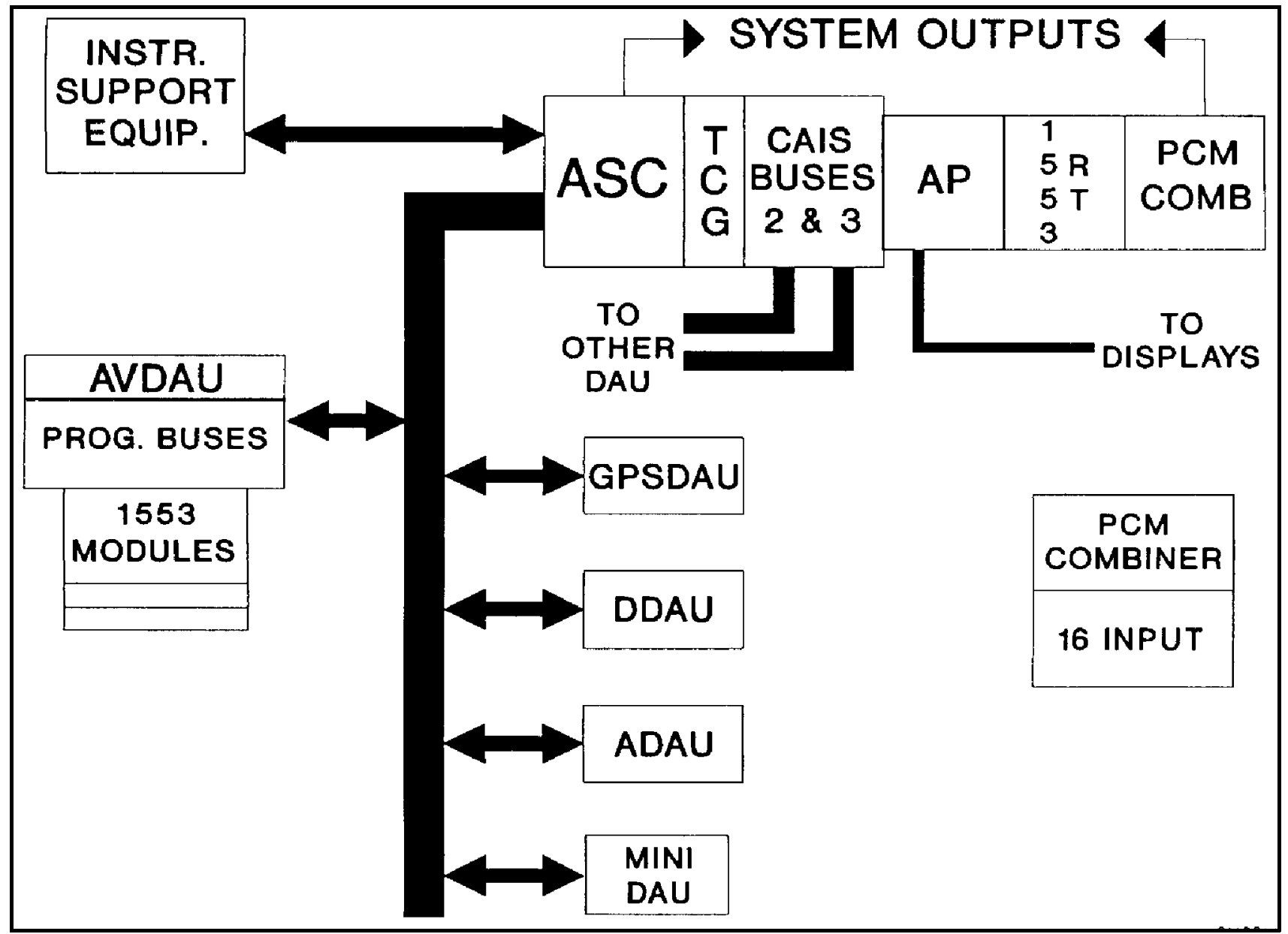

Figure 2 


\section{GROUND SEGMENT}

Portable Flight-line Unit (PFU)

The PFU provides the capability to generate formats, load/verify airborne memories, perform data limit check and to look at raw data. The PFU is primarily an IBM compatible portable personal computer with the CAIS ground support software and a few interface boards. The software will perform most functions without the presence of the interface cards to allow the user to develop test configurations and format loads on a desktop computer.

\section{Hand Held Decom (HHD)}

The hand held decom is a shoebox size decommutator. It requires a PCM data stream with the associated framing signals (i.e. bit clock, word clock, minor frame clock and major frame clock). The HHD has two independent numeric displays with a binary display under each. The two numeric displays are selectable between decimal and hexadecimal.

\section{CAIS Bus Decom (CBD)}

The CAIS Bus Decom is a hand held unit the same size as the HHD. The CBD serves as a CAIS bus monitor. The CBD displays the replies corresponding to a command word number dialed in on the thumbwheel switches.

\section{Analog Signal Generator (ASG)}

The analog signal generator is a suitcase sized unit which will stimulate all of the analog signal conditioning cards of the ADAU and some of the digital ones too. The ASG is meant to provide an efficient way of doing an end to end check of the system installed in the aircraft.

\section{DEVELOPMENT SCHEDULE}

Figure 3 presents an overview of the CAIS full scale development schedule. Hardware being produced include an engineering model and several sets of flight demonstration units. The flight demonstration units will be fully qualified and used by the services to perform a series of CAIS flight tests prior to actual fielding of the system.

By the conclusion of the development program a complete set of documentation including drawings, manuals, specifications, and software documentation in accordance with DOD-STD-2167A are to be delivered to the government. The government will use this documentation to provide life cycle support for CAIS and to competitively reprocure identical units to satisfy the flight test needs of the services in the coming years. 


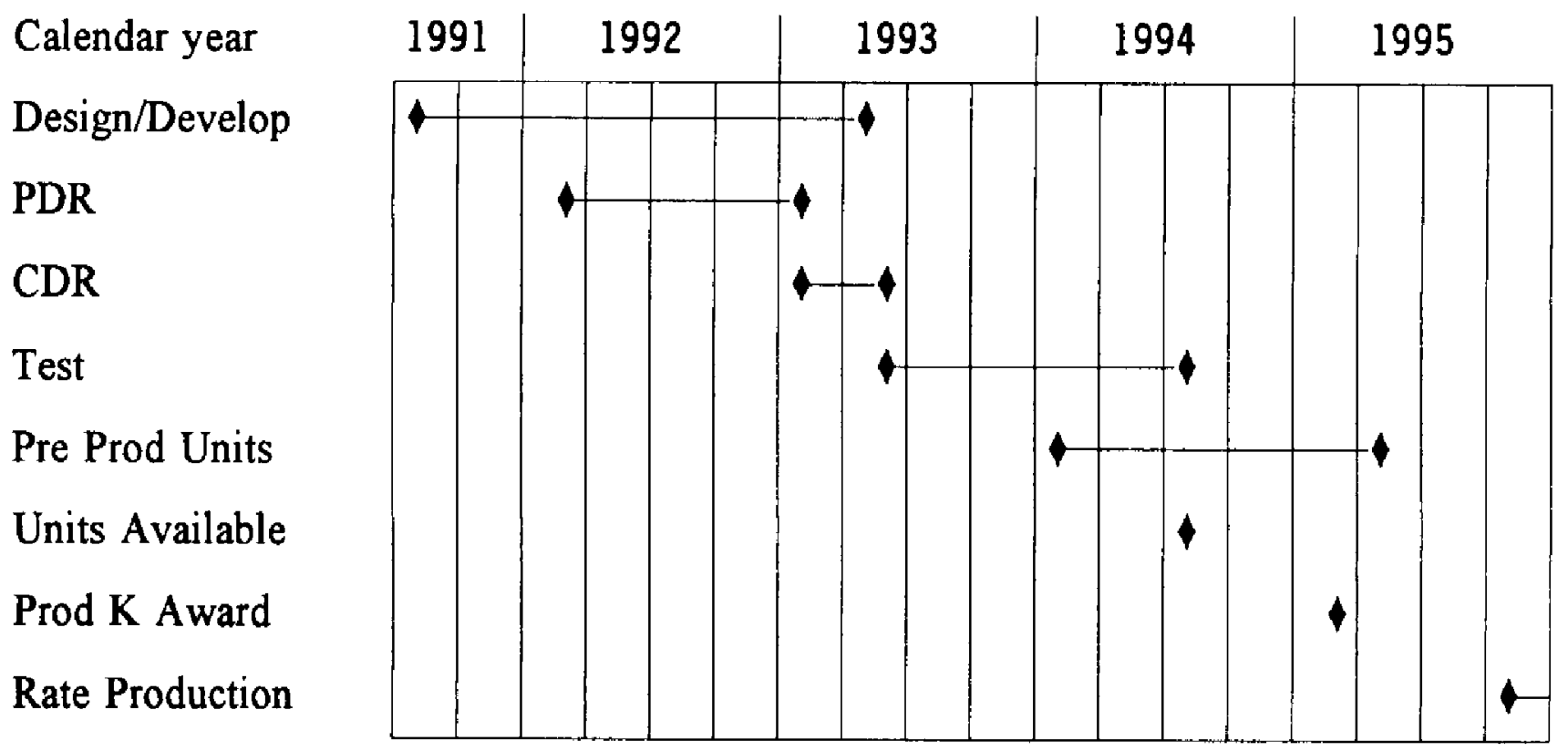

Figure 3. CAIS Development Schedule 